Ciência Florestal, Santa Maria, v. 27, n. 1, p. 277-290, jan.-mar., 2017

ISSN 1980-5098

\title{
AVALIAÇÃO SILVICULTURAL DE DEZ ESPÉCIES NATIVAS DA MATA ATLÂNTICA
}

\author{
SILVICULTURAL EVALUATION OF TEN NATIVE SPECIES OF THE ATLANTIC FOREST
}

\author{
Guilherme Carneiro de Mendonça ${ }^{1}$ José Franklim Chichorro ${ }^{2}$ Adriano Ribeiro de Mendonça ${ }^{3}$ \\ Lorena Abdalla de Oliveira Prata Guimarães ${ }^{4}$
}

\begin{abstract}
RESUMO
Com a previsão de escassez de recursos florestais amazônicos é eminente a necessidade de estudos sobre o potencial silvicultural de espécies de outras regiões como a Mata Atlântica. O objetivo deste estudo foi avaliar características silviculturais das espécies Astronium concinnum, Cariniana legalis, Goniorrhachis marginata, Kielmeyera albopunctata, Lecythis pisonis, Manilkara bella, Basiloxylon brasiliensis, Spondias venulosa, Vitex sp. e Zeyheria tuberculosa, nativas da Mata Atlântica com ocorrência no Espírito Santo. As espécies foram plantadas a pleno sol em plantios puros, com espaçamento de 2 x 2 $\mathrm{m}$. Os povoamentos possuíam idades entre 21 e 25 anos na época da avaliação final e foram conduzidos na Reserva Natural da Vale, nos municípios de Linhares e Sooretama, Espírito Santo. Foram avaliados falha de plantio, fitossanidade, quebra, bifurcação, forma do fuste, diâmetro a altura do peito, altura total e incremento médio anual volumétrico. O volume foi estimado por meio de diferentes equações ajustadas para cada espécie. Constataram-se falhas de plantio expressivas apenas para Cariniana legalis, Zeyheria tuberculosa e Goniorrhachis marginata. Apenas Vitex sp. apresentou restrições fitossanitárias. Spondias venulosa, Goniorrhachis marginata e Kielmeyera albopunctata destacaram-se com os maiores índices de quebras. Goniorrhachis marginata, Manilkara bella e Basiloxylon brasiliensis destacaramse com os menores índices de bifurcação. Manilkara bella, Cariniana legalis e Zeyheria tuberculosa destacaram-se quanto à forma de fuste retilíneo. Spondias venulosa apresentou crescimento expressivo e superior às demais espécies. São necessários mais estudos para subsidiar o reflorestamento econômico dessas espécies, destacando-se estudos de melhoramento genético, técnicas silviculturais, técnicas de desdobro e de tecnologia da madeira que apontem as aptidões de uso.
\end{abstract}

Palavras-chave: produção madeireira; recursos florestais; manejo florestal.

\section{ABSTRACT}

Along with the forecast shortage of the Amazonian forest resources is imminent the need for studies concerning the silvicultural potential of species from other regions like the Atlantic Forest. The purpose of this study was to evaluate the silvicultural characteristics of Astronium concinnum, Cariniana legalis, Goniorrhachis marginata, Kielmeyera albopunctata, Lecythi pisonis, Manilkara bella, Basiloxylon brasiliensis, Spondias venulosa, Vitex sp. and Zeyheria tuberculosa, native Atlantic Forest species occurring in Espírito Santo state. The species were planted in full sun in pure stands, with spacing of $2 \times 2 \mathrm{~m}$. The

1 Engenheiro Florestal, MSc., Agente de Desenvolvimento Ambiental e Recursos Hídricos, Instituto Estadual de Meio Ambiente e Recursos Hídricos - IEMA, BR-262, Km 90, Pedra Azul, CEP 29278-000, Domingos Martins (ES), Brasil. gmendonca@iema.es.gov.br

2 Engenheiro Florestal, Dr., Professor Associado do Departamento de Ciências Florestais e da Madeira, Universidade Federal do Espírito Santo, Av. Governador Lindemberg, 316, Centro, CEP 29550-000, Jerônimo Monteiro (ES), Brasil.jfufes@gmail.com

3 Engenheiro Florestal, Dr., Professor Adjunto do Departamento de Ciências Florestais e da Madeira, Universidade Federal do Espírito Santo, Avenida Governador Lindemberg, 316, Centro, CEP 29550-000, Jerônimo Monteiro (ES), Brasil. adriano.mendonca@ufes.br

4 Engenheira Agrônoma, Dra ${ }^{\mathrm{a}}$, Agente de Pesquisa e Inovação em Desenvolvimento Rural, Instituto Capixaba de Pesquisa, Assistência Técnica e Extensão Rural - Incaper, BR 262, km 94, Fazenda do Estado, CEP 29375-000, Venda Nova do Imigrante (ES), Brasil. lorena.prata@hotmail.com

Recebido para publicação em 11/08/2014 e aceito em 14/04/2015

Ci. Fl., v. 27, n. 1, jan.-mar., 2017 
stands had ages between 21 and 25 at the time of final evaluation and were conducted at Vale Nature Reserve, in the municipalities of Sooretama and Linhares, ES state. The variables collected and analyzed annually were: failure of plant, plant health, breaking, forking, stem form, diameter at breast height, total height and volumetric average annual increment. The volume was estimated using models developed for each species. It was verified expressive faults planting only Cariniana legalis, Zeyheria tuberculosa and Goniorrhachis marginata. Only Vitex sp. presented phytosanitary restrictions. Spondias venulosa, Goniorrhachis marginata and Kielmeyera albopunctata stood out with the highest rates of breakage. Goniorrhachis marginata, Manilkara bella and Basiloxylon brasiliensis stood out with the lowest levels of bifurcation. Manilkara bella, Cariniana legalis e Zeyheria tuberculosa stood out as the form of a straight stem form. Spondias venulosa presented an expressive and superior to other species growth. More studies are needed to support the economic reforestation of that species, emphasis on studies of genetic improvement, silvicultural techniques, techniques of sawing and timber technology that points out the skills to use.

Keywords: timber production; forest resources; forest management.

\section{INTRODUÇÃO}

Apesar da importância da biodiversidade e dos serviços ambientais prestados pela Mata Atlântica para a maioria da população brasileira, monitoramentos recentes indicam que suas florestas ainda passam por um processo contínuo de desmatamento e fragmentação (HIROTA, 2013). A drástica redução da área de ocupação desse bioma confirma sua fragilidade e compromete sua biodiversidade.

Para a efetiva conservação da Mata Atlântica, produtores rurais devem ser envolvidos e estimulados a adotarem práticas sustentáveis em suas propriedades, gerando benefícios ambientais e econômicos diretos. Para isso, estudos sobre as características silviculturais das espécies nativas devem ser realizados com a finalidade de gerar conhecimento e possibilitar o desenvolvimento de tecnologias de cultivo.

As tecnologias silviculturais amplamente desenvolvidas nas últimas décadas restringem-se a poucas espécies arbóreas, notadamente exóticas. Na região sudeste, em especial, esse desenvolvimento se restringe às espécies dos gêneros Pinus e Eucalyptus. A preferência pelo cultivo de espécies exóticas deve-se, dentre outros fatores, à falta de conhecimento técnico sobre o manejo de espécies nativas e ao direcionamento do poder público, que investiu em pesquisa e desenvolvimento tecnológico e induziu, por meio de incentivos fiscais, o plantio de espécies exóticas (ASSOCIAÇÃO BRASILEIRADE PRODUTORES DE FLORESTAS PLANTADAS, 2012; BRANCALION et al., 2012; YOUNG, 2003). O incentivo e o desenvolvimento silvicultural restritos ao cultivo de espécies exóticas não só subutilizam o potencial de mercado de muitas espécies nativas, muitas das quais raras em paisagens rurais devido à exploração irracional no passado recente, mas limitam também a conservação e o resgate genético das mesmas, mantendo a condição de extinção local de muitas espécies.

Não é comum na literatura científica brasileira estudos de avaliação silvicultural de espécies nativas da Mata Atlântica. Quando estudos sobre essas espécies são publicados, geralmente se referem a levantamentos florísticos e fitossociológicos e a componentes químicos de suas estruturas. Dados de crescimento, quando encontrados, estão relacionados, em sua maioria, a trabalhos de recuperação ambiental. Os dados silviculturais mais relevantes podem ser encontrados nas publicações de Carvalho (2003; 2006; 2010).

Para melhor aproveitamento das características edafoclimáticas favoráveis e do potencial de muitas espécies nativas para a silvicultura, é imprescindível que se conheça o comportamento silvicultural dessas espécies em plantios experimentais. Esse conhecimento possibilitará o desenvolvimento de programas mais amplos de cultivo dessas espécies envolvendo atividades essenciais para sua silvicultura como seleção genética, desenvolvimento de modelos de reflorestamento e de tecnologias de produção de multiprodutos da floresta. Ressalta-se, ainda, que, além de retornos econômicos, seria possível resgatar alguns dos serviços ambientais prestados pelas florestas naturais.

Em geral, os estudos de potencial silvicultural de espécies nativas dão ênfase às características de crescimento das plantas em diâmetro, altura e volume. É conhecimento comum que essas características são de grande importância em estudos de potencial silvicultural. No entanto, outras, igualmente relevantes para a recomendação de uso de uma espécie ou que indiquem a qualidade da madeira e o seu potencial de 
uso, são negligenciadas em muitos estudos, a exemplo da taxa de sobrevivência, fitossanidade, tortuosidade e ramificação do tronco.

Desse modo, o objetivo do presente estudo foi avaliar características silviculturais de dez espécies aarbóreas nativas da Mata Atlântica, plantadas na forma de povoamentos puros, pretendendo-se, assim, ampliar o conhecimento das mesmas e as possibilidades de uso.

\section{MATERIAL E MÉTODO}

\section{Caracterização da área de estudo}

O estudo foi realizado na Reserva Natural Vale (RNV), situada entre os municípios de Linhares e Sooretama, no estado do Espírito Santo. As áreas dos povoamentos estudados pertencem à formação denominada Tabuleiros Costeiros, cujo relevo varia de plano a suave ondulado. A altitude da área varia de 50 a 60 metros. A vegetação nativa da região é classificada como Floresta Ombrófila densa de terras baixas (VELOSO et al., 1991).

Os solos da região são classificados como Argissolo Amarelo distrófico (KINDEL; GARAY, 2001). Segundo Garay et al., (1995), esses solos apresentam normalmente horizonte A moderado e horizonte B textural e uma típica pobreza química do solo.

Segundo a classificação de Köppen, o clima da região é do tipo Awi, sendo quente e úmido, com estação chuvosa no verão e seca no inverno. Dados médios da série histórica indicam que a precipitação média anual é de $1.283 \mathrm{~mm}$ (INSTITUTO CAPIXABA DE PESQUISA, ASSISTÊNCIA E EXTENSÃO RURAL, 2012). A temperatura média anual é de $24,5^{\circ} \mathrm{C}$, com máxima de $32^{\circ} \mathrm{C}$ e mínima de $17^{\circ} \mathrm{C}$. A umidade média relativa do ar é de $83 \%$. A velocidade média dos ventos, a 50 metros de altura, é em torno de $5 \mathrm{~m}$ $\mathrm{s}^{-1}$ (AGÊNCIA DE SERVIÇOS PÚBLICOS DE ENERGIA DO ESTADO DO ESPÍRITO SANTO, 2012).

\section{Descrição dos povoamentos}

As espécies avaliadas (Tabela 1) foram plantadas em povoamentos puros a pleno sol. Cada povoamento foi implantado em uma parcela de observação de $784 \mathrm{~m}^{2}$, com espaçamento de $2 \times 2$ metros, totalizando 196 indivíduos. Apenas o povoamento de Basiloxylon brasiliensis foi implantado em uma área de $588 \mathrm{~m}^{2}$, com 147 indivíduos.

\section{Avaliação dos povoamentos}

As características de cada indivíduo dos povoamentos foram observadas anualmente desde a implantação. Foram avaliadas cinco características qualitativas (Tabela 2), levantadas visualmente, e três características dendrométricas - diâmetro a altura do peito (DAP), determinado a 1,30 m do solo, altura total (H) e incremento médio anual volumétrico (IMAv).

A característica falha de plantio foi avaliada indicando o percentual de plantas ou mudas mortas durante os três primeiros anos de plantio. Registros de perdas posteriores foram considerados como mortalidade por outros fatores que não o plantio.

As avaliações de fitossanidade não envolveram a identificação da praga ou do patógeno causador de danos. Nesse caso, foi avaliado apenas o grau de dano causado em cada indivíduo por pragas ou doenças.

Quanto à suscetibilidade à quebra, as plantas com danos no fuste e em suas bifurcações foram classificadas como árvores quebradas. Para a característica bifurcação, os indivíduos foram classificados em árvore bifurcada ou normal, avaliando-se apenas os indivíduos que apresentaram ou não multitroncos ou polifurquia. Portanto, não foi avaliada a intensidade de ramificação.

Para a avaliação da forma de fuste, utilizou-se como referência, o ano em que ocorreu o maior número de indivíduos nas piores condições (tortuoso ou malformado). Dessa forma, essa característica foi obtida no oitavo ano de idade do povoamento de Zeyheria tuberculosa, no nono de Spondias venulosa, no décimo de Manilkara bella, no décimo primeiro de Astronium concinnum e Cariniana legalis, no décimo segundo de Vitex sp. e Basiloxylon brasiliensis, no décimo terceiro de Goniorrhachis marginata, no décimo 
TABELA 1: Caracterização dos povoamentos das espécies arbóreas nativas da Mata Atlântica implantadas em plantios puros, no espaçamento de 2 × 2 metros em Linhares - ES.

TABLE 1: Characterization of stands of native tree species of the Atlantic Forest implemented in pure stands with spacing of $2 \times 2$ meters in Linhares, ES state.

\begin{tabular}{|c|c|c|c|}
\hline Espécie & Implantação & Idade $^{1}$ & Tratos silviculturais \\
\hline $\begin{array}{l}\text { Astronium concinnum Schott - } \\
\text { gonçalo-alves, guaribu-preto }\end{array}$ & nov./87 & 22 & \multirow{3}{*}{$\begin{array}{l}\text { Adubação: } 200 \mathrm{~g} \text { de superfosfato simples por } \\
\text { cova no plantio, } 20 \mathrm{~g} \text { de cloreto de potássio e } 30 \mathrm{~g} \\
\text { de sulfato de amônio em cobertura, aos } 120 \text { dias. } \\
\text { Controle sistemático de gramíneas. Desbastes e } \\
\text { desramas circunstanciais. }\end{array}$} \\
\hline $\begin{array}{l}\text { Cariniana legalis (Mart) Kuntze. } \\
\text { - jequitibá-rosa }\end{array}$ & nov./87 & 22 & \\
\hline $\begin{array}{l}\text { Kielmeyera albopunctata Saddi. } \\
\text { - nagibe }\end{array}$ & nov./87 & 23 & \\
\hline $\begin{array}{l}\text { Lecythis pisonis Cambess. - } \\
\text { sapucaia-vermelha }\end{array}$ & mar./88 & 22 & \multirow{4}{*}{$\begin{array}{l}\text { Adubação: } 200 \mathrm{~g} \text { de superfosfato simples por } \\
\text { cova no plantio, } 20 \mathrm{~g} \text { de cloreto de potássio e } 30 \mathrm{~g} \\
\text { de sulfato de amônio em cobertura, aos } 120 \text { dias. } \\
\text { Controle sistemático de gramíneas. Desbastes e } \\
\text { desramas circunstanciais. }\end{array}$} \\
\hline $\begin{array}{l}\text { Manilkara bella Monach. - } \\
\text { paraju, maçaranduba-vermelha }\end{array}$ & nov./87 & 22 & \\
\hline Vitex sp. - tarumã & nov./87 & 23 & \\
\hline $\begin{array}{l}\text { Zeyheria tuberculosa } \text { (Vell) } \\
\text { Bureau ex Verl. - ipê-felpudo }\end{array}$ & nov./87 & 22 & \\
\hline $\begin{array}{l}\text { Spondias venulosa (Engl) Engl. - } \\
\text { cajá-nativo, cajá-grande }\end{array}$ & dez. $/ 88$ & 21 & \multirow{2}{*}{$\begin{array}{l}\text { Adubação: } 200 \mathrm{~g} \text { de superfosfato simples por cova } \\
\text { no plantio. Controle sistemático de gramíneas. } \\
\text { Desbastes e desramas circunstanciais. }\end{array}$} \\
\hline $\begin{array}{l}\text { Goniorrhachis marginata Taub. - } \\
\text { guaribu-amarelo, itapicuru }\end{array}$ & mar./89 & 21 & \\
\hline $\begin{array}{l}\text { Basiloxylon brasiliensis } \\
\text { (Allemão) K. Schum - farinha- } \\
\text { seca }\end{array}$ & set./83 & 25 & $\begin{array}{c}\text { Adubação: } 250 \mathrm{~kg} / \mathrm{ha} \text { de superfosfato simples no } \\
\text { plantio e } 60 \mathrm{~kg} / \mathrm{ha} \text { de cloreto de potássio e } 100 \mathrm{~kg} / \\
\text { ha de sulfato de amônio em cobertura, aos } 60 \mathrm{dias} \text {. } \\
\text { Controle sistemático de gramíneas. Desbastes } \\
\text { circunstanciais. }\end{array}$ \\
\hline
\end{tabular}

Em que: ${ }^{1}$ Idade do povoamento, em anos, no último ano de avaliação.

quarto de Lecythis pisonis e no décimo quinto ano de Kielmeyera albopunctata. Assim, o percentual de indivíduos de cada classificação referiu-se apenas a essas idades. Essa estratégia teve que ser utilizada devido aos desbastes circunstanciais que eram direcionados aos indivíduos mais tortuosos.

As características qualitativas foram analisadas de forma descritiva, buscando-se identificar a relação entre os resultados encontrados e as características ecológicas das espécies e as técnicas silviculturais aplicadas.

O IMAv foi estimado por meio da aplicação de modelos volumétricos que tiveram suas equações ajustadas por Martins (2012), em estudo realizado nos mesmos povoamentos. Os modelos utilizados foram Ogaya (OGAYA, 1968) para Astronium concinnum, Kielmeyera albopunctata, Zeyheria tuberculosa e Basiloxylon brasiliensis; Stoat (STOAT, 1945) para Lecythis pisonis e Manilkara bella; Spurr linear (SPURR, 1952) para Vitex sp. e Cariniana legalis; e Schumacher e Hall (SCHUMACHER; HALL, 1933) para Spondias venulosa e Goniorrhachis marginata.

Os valores médios de DAP, H e IMAv das espécies foram comparados estatisticamente pelo teste $t$. A análise foi realizada somente com os dados obtidos aos 21 anos de idade dos povoamentos, maior idade na qual todos os povoamentos foram avaliados. Apenas os povoamentos que apresentaram distribuição normal para as referidas variáveis foram comparados pelo teste $t$, sendo a normalidade dos dados verificada pelo teste de Lilliefors. 
TABELA 2: Características qualitativas avaliadas nos povoamentos das espécies arbóreas nativas da Mata Atlântica implantadas em plantios puros, no espaçamento de 2 × 2 metros em Linhares - ES, e suas respectivas classificações.

TABLE 2: Qualitative characteristics evaluated of stands of native tree species of the Atlantic Forest implemented in pure stands with spacing of $2 \times 2$ meters in Linhares, ES state, and their respective classifications.

\begin{tabular}{lc}
\hline \multicolumn{1}{c}{ Característica } & Classificação \\
\hline \multirow{2}{*}{ Falha de plantio } & Planta morta \\
& Planta viva \\
& Árvore sadia \\
Fitossanidade & Árvore pouco doente (25 a $50 \%$ da árvore danificada) \\
& Árvore muito doente (50 a $100 \%$ da árvore danificada) \\
Suscetibilidade à quebra & Árvore morta \\
& Árvore quebrada \\
Bifurcação & Árvore normal \\
& Árvore bifurcada \\
& Árvore normal \\
Forma do fuste & Retilíneo \\
& Levemente tortuoso \\
& Tortuoso \\
& Malformado \\
\hline
\end{tabular}

\section{RESULTADOS E DISCUSSÃO}

\section{Sobrevivência}

Não foram verificadas expressivas falhas de plantio para a maioria das espécies estudadas (Tabela 3). Apenas três espécies apresentaram valores superiores a 5\%, com destaque para Cariniana legalis. Essa observação corrobora as informações da literatura sobre as espécies, que as classificam como potenciais heliófilas, com exceção de Cariniana legalis.

Segundo Oberbauer et al. (1993), citados por Tanaka e Vieira (2006), espécies tolerantes à sombra são menos hábeis para se adaptar a locais abertos. Assim, Cariniana legalis pode ter apresentado restrições ao plantio a pleno sol, pois essa espécie é classificada como secundária tardia e semi-heliófila, que não regenera naturalmente em áreas abertas (CARVALHO, 2003).

O elevado percentual de falhas de plantio observado no povoamento de Zeyheria tuberculosa não era esperado, uma vez que essa espécie possui plasticidade edafoclimática e capacidade de colonizar pastagens degradadas (CARVALHO, 2003). Sua adaptabilidade a diferentes condições de luz foi confirmada por Engel e Poggiani (1990), que registraram índices de sobrevivência de 96,3\% a 100\%. Carvalho (2003), porém, registrou valores de falhas de plantio para essa espécie que variaram de $6,3 \%$ a $30,5 \%$. O elevado percentual de falha indica que mesmo para algumas espécies tidas como ruderais, o desenvolvimento de tratamentos silviculturais adequados são fundamentais para o seu melhor desempenho em plantios.

Apesar de a falha de plantio ser um importante critério de avaliação silvicultural, ela não pode encerrar uma análise da capacidade de adaptação de uma espécie em um sítio. De acordo com Andrade (1991), a taxa de mortalidade pode ser decorrente não só da adaptabilidade da espécie, mas também de falhas técnicas na produção e plantio das mudas, de incêndios, de ataques localizados de formigas cortadeiras ou de outras pragas e doenças. 
TABELA 3: Percentual de indivíduos (\%) encontrados em cada classificação das características qualitativas avaliadas de espécies arbóreas nativas da Mata Atlântica em plantios puros, no espaçamento de 2 × 2 metros em Linhares - ES.

TABLE 3: Percentage of individuals (\%) found in every classification of the qualitative characteristics evaluated of native tree species of the Atlantic Forest in pure stands with spacing of $2 \times 2$ meters in Linhares, ES state.

\begin{tabular}{|c|c|c|c|c|c|}
\hline \multirow{2}{*}{ Espécie } & \multicolumn{2}{|l|}{ Sobrevivência } & \multicolumn{2}{|l|}{ Fitossanidade } & \multirow{2}{*}{$\begin{array}{c}\text { Susceptibilidade } \\
\text { à quebra } \\
\text { Quebrado }\end{array}$} \\
\hline & Falha de plantio & $\begin{array}{l}\text { Pouco } \\
\text { doente }\end{array}$ & Muito doente & Morto & \\
\hline Astronium concinnum & 1 & 10 & 1 & 0 & 9 \\
\hline Cariniana legalis & 20 & 2 & 0 & 0 & 7 \\
\hline Kielmeyera albopunctata & 2 & 1 & 0 & 0 & 22 \\
\hline Lecythis pisonis & 0 & 3 & 0 & 0 & 6 \\
\hline Manilkara bella & 3 & 1 & 0 & 0 & 11 \\
\hline Vitex sp. & 0 & 19 & 7 & 0 & 19 \\
\hline Zeyheria tuberculosa & 14 & 2 & 1 & 0 & 17 \\
\hline Spondias venulosa & 2 & 5 & 1 & 0 & 30 \\
\hline Goniorrhachis marginata & 12 & 5 & 1 & 1 & 26 \\
\hline Basiloxylon brasiliensis & 3 & 1 & 2 & 0 & 17 \\
\hline \multirow[b]{2}{*}{ Espécie } & Bifurcação & \multicolumn{4}{|c|}{ Forma de fuste } \\
\hline & Bifurcado & Retilíneo & $\begin{array}{c}\text { Levemente } \\
\text { tortuoso }\end{array}$ & Tortuoso & Malformado \\
\hline Astronium concinnum & 88 & 0 & 96 & 3 & 1 \\
\hline Cariniana legalis & 34 & 8 & 83 & 3 & 6 \\
\hline Kielmeyera albopunctata & 84 & 0 & 79 & 21 & 0 \\
\hline Lecythis pisonis & 97 & 0 & 99 & 1 & 0 \\
\hline Manilkara bella & 5 & 9 & 90 & 0 & 1 \\
\hline Vitex sp. & 95 & 0 & 59 & 41 & 0 \\
\hline Zeyheria tuberculosa & 22 & 6 & 84 & 7 & 3 \\
\hline Spondias venulosa & 97 & 0 & 1 & 99 & 0 \\
\hline Goniorrhachis marginata & 0 & 0 & 63 & 37 & 0 \\
\hline Basiloxylon brasiliensis & 18 & 2 & 96 & 2 & 0 \\
\hline
\end{tabular}

\section{Fitossanidade}

De modo geral, as espécies não apresentavam problemas de fitossanidade e as mortes provenientes de pragas ou doenças foram muito baixas em todos os povoamentos, com exceção de Vitex sp. Esse resultado indica que, para plantios puros nas mesmas condições, Vitex sp. requer cuidados fitossanitários. Se plantada em povoamentos mistos, a cautela deve ser no sentido de evitar que espécies suscetíveis a pragas e doenças se tornem um fator de atração de insetos e patógenos.

É possível que o regime de desbastes tenha minimizado a proliferação de pragas e doenças nos povoamentos, como o observado para o povoamento de Vitex sp., no qual foi identificada a concentração dos desbastes nos indivíduos mais doentes. 


\section{Suscetibilidade à quebra}

Observou-se que os maiores percentuais de quebra tenderam a se concentrar nas espécies que possuem madeiras com menores valores de densidade (CARVALHO, 2003; 2006; 2010; GONZAGA, 2006; INSTITUTO DE PESQUISAS TECNOLÓGICAS DO ESTADO DE SÃO PAULO, 2012; LORENZI, 2008; 2009; IBAMA, 2012), em Goniorrhachis marginata, entretanto, não seguiu essa tendência. Dentre as espécies avaliadas, esta possui o segundo maior valor de densidade média da madeira e, ainda assim, apresentou o segundo maior percentual de indivíduos quebrados.

A diferença na suscetibilidade à quebra de Spondias venulosa e de Basiloxylon brasiliensis, que possuem as madeiras de mais baixa densidade e são tipicamente da fase inicial de sucessão, provavelmente, deve-se aos diferentes padrões alométricos e às estratégias de ocupação em espécies arbóreas pioneiras, evidenciados por Fontes (1999). Essas diferenças são evidentes ao se observar que Spondias venulosa apresentou elevado índice de bifurcação e tortuosidade do tronco, enquanto que Basiloxylon brasiliensis apresentou baixos índices de bifurcação e tortuosidade.

É possível que, além de fatores genéticos e externos, como a força dos ventos, algumas práticas silviculturais adotadas, como o reduzido espaçamento inicial e os desbastes, tenham influenciado a quebra do fuste das árvores avaliadas. Em se tratando dos desbastes, sua influência pode ter sido por meio de danos causados durante as derrubadas e pela abertura de espaços que possibilitaram a entrada de maiores correntes de vento. Martins et al. (1997), avaliando o impacto da exploração de madeira em florestas naturais, observaram que, em média, 22,9 \% do número total de árvores por hectare foram severamente danificadas.

A influência dos desbastes foi mais evidente quando se verificou que as idades de máxima ocorrência de quebra foram próximas das idades de primeiro desbaste intenso, por volta de seis e sete anos. Um exemplo é o Vitex sp., que apresentou elevado índice de quebra e passou por quatorze intervenções de desbastes. É possível que a adoção de desbastes seletivos nesses povoamentos de alta densidade de plantas, ao invés de sistemáticos ou mistos, possa ter influenciado nos percentuais de quebra obtidos.

\section{Bifurcação}

Goniorrhachis marginata foi a espécie que apresentou o menor percentual de indivíduos com bifurcação de fuste. Apesar de não haver relatos de avaliação desta espécie em outros plantios, além de fatores genéticos é possível que o espaçamento reduzido possa ter influenciado na ausência de bifurcação nos indivíduos dessa espécie.

O comportamento de Vitex sp. concorda com o descrito na literatura para Vitex megapotâmica (CARVALHO, 2006), apresentando ramificação dicotômica e copa irregular.

Apenas no povoamento de Cariniana legalis foram verificadas quantidades medianas de indivíduos bifurcados, indicando que a característica bifurcação, quando manifestada, tende a ocorrer na maior parte dos indivíduos dos povoamentos dessas espécies quando plantadas na mesma condição.

Um aspecto importante e que deve ser considerado é a altura de ocorrência da bifurcação ou o comprimento do fuste comercial. A diferença entre a altura total da planta e a altura do fuste comercial indica possíveis perdas de rendimento no processamento e/ou na qualidade da madeira (MATTOS et al., 2003). Nas análises visuais de campo, observou-se que as plantas de Astronium concinnum, Cariniana legalis e Basiloxylon brasiliensis apresentaram ramificações na parte mais alta de seus troncos e, portanto, fustes comerciais mais extensos. Essa característica reduz possíveis perdas de rendimento no processamento como, por exemplo, para Astronium concinnum, que apresentou um dos maiores percentuais de indivíduos bifurcados.

O elevado índice de árvores bifurcadas encontrado para Lecythis pisonis corrobora as informações da literatura, que a cita como espécie de forma irregular, sem dominância apical definida e ramificação pesada sem desrama natural (CARVALHO, 2006). Contudo, a observação de indivíduos de Lecythis pisonis remanescentes de antigas florestas indica que esta espécie, quando se desenvolve à sombra ou com competição lateral, pode não manifestar a ramificação encontrada quando plantada a pleno sol. Alguns autores citam espécies que apresentam comportamento semelhante, com tronco longo e livre de galhos em plantio consorciado com uma espécie de mais rápido crescimento e tronco curto e engalhado em plantios 
puros (CARVALHO, 2010; KAGEYAMA; CASTRO, 1989). Schilling et al. (1998) afirmam ainda que a ramificação é uma característica de menor herdabilidade, sendo, portanto, muito mais afetada pelo ambiente ou pelo espaço disponível para desenvolvimento do que por fatores genéticos.

Segundo Whatley e Whatley (1982), citados por Engel e Poggiani (1990), a capacidade de desenvolvimento das espécies em diferentes graus de sombreamento é importante principalmente na fase juvenil por condicionar mudanças morfogenéticas e fisiológicas. Kageyama e Castro (1989) destacam que o conhecimento da ecologia das espécies florestais é muito importante, pois estas possuem ritmos de crescimento e necessidades ecológicas diferentes nos diversos estágios de desenvolvimento.

Percebe-se, assim, que é muito importante o conhecimento da ecofisiologia das espécies para o planejamento de projetos de povoamentos comerciais. Uma mesma espécie que apresenta baixo potencial silvicultural para um determinado fim em um sistema de plantio pode responder melhor em relação a algumas características em outro sistema (por exemplo, em plantios homogêneos ou em plantios mistos com espécies de ritmos distintos de crescimento). Além disso, para povoamentos comerciais com produção voltada para uso em serraria, a possibilidade de ganhos de rendimento no processamento do fuste e a redução da necessidade de se efetuar a desrama podem determinar um significativo e positivo impacto econômico.

\section{Forma do fuste}

De maneira geral, houve predomínio de fustes levemente tortuosos, com exceção de Spondias venulosa, que apresentou predominância de fustes tortuosos. Isso indica que as espécies apresentaram boas condições de fuste para produção de madeira voltada para serraria.

O povoamento de Cariniana legalis foi o que apresentou o maior índice de árvores malformadas, o que pode estar relacionado com o elevado índice de falha de plantio. Os resultados indicam que Cariniana legalis pode ter tido problemas adaptativos na condição em que foi plantada ou ainda que pode ter ocorrido problemas na qualidade das mudas ou no procedimento de plantio. Para essa espécie, Silva (2013) observou fustes retos e sem ramificações em reflorestamentos mistos, nos quais os indivíduos cresceram com sombreamento parcial.

É provável que a alta densidade inicial de plantio (2.500 plantas ha $\left.{ }^{-1}\right)$ tenha influenciado na reduzida tortuosidade da maioria das espécies. Além disso, é possível que a subjetividade intrínseca do tipo de avaliação (avaliação visual), o número relativamente alto de categorias de classificação (4) e o rigor nas avaliações de campo tenham resultado na baixa incidência de fustes retilíneos. Todos esses fatores podem ter contribuído para a elevada frequência de fustes levemente tortuosos.

\section{Dendrometria}

Spondias venulosa apresentou o DAP significativamente superior ao das demais espécies avaliadas aos 21 anos de idade (Tabela 4). O IMAv e a $\mathrm{H}$ dessa espécie também foram superiores ao das demais em valores absolutos. No entanto, não foi possível verificar se essa diferença foi significativa estatisticamente.

Contudo, apesar do destacado crescimento volumétrico, essa espécie pode apresentar limitações para a utilização em serraria, pois, nas condições avaliadas, o povoamento apresentou elevado percentual de indivíduos bifurcados (97\%) e tortuosos (99\%). Em se tratando da tortuosidade, há grande perda de rendimento no processamento da madeira, o que pode ser compensando pelo rápido crescimento da espécie. Segundo Justiniano et al. (2001), a madeira dessa espécie pode ser usada para compensados, carpintaria interior, molduras, embalagens e aeromodelismo, além de outros usos.

Segundo Marchesan (2012) e Rocha (2002), a dimensão da tora é um dos principais fatores que afetam o rendimento volumétrico no processamento. Considerando um DAP mínimo para utilização em serraria de $15 \mathrm{~cm}$, Spondias venulosa, com apenas seis anos de formação, já apresentou DAP médio superior a esse valor (Figura 1). Aos 21 anos de idade, o DAP médio do povoamento chegou a $32,3 \mathrm{~cm}$.

Os povoamentos de Goniorrhachis marginata, Vitex sp. e Manilkara bella, mesmo em idades superiores a 20 anos, produziram toras com DAP médio inferior a $15 \mathrm{~cm}$ (Figura 2). Por essa razão, o plantio dessas espécies para a utilização em serraria só será economicamente viável se for obtido alto valor 
TABELA 4: Valores médios de diâmetro a altura do peito (DAP), altura total $(\mathrm{H})$ e incremento médio anual volumétrico (IMAv) de espécies arbóreas nativas da Mata Atlântica em plantios puros, no espaçamento de 2 × 2 metros em Linhares - ES, aos 21 anos de idade.

TABLE 4: Mean values of diameter at breast height (DAP), total height $(\mathrm{H})$ and volumetric mean annual increment (IMAv) of native tree species of the Atlantic Forest in pure stands with spacing of 2 x 2 meters in Linhares, ES state, at 21 years old.

\begin{tabular}{lccc}
\hline \multicolumn{1}{c}{ Espécie } & $\mathrm{DAP}(\mathrm{cm})$ & $\mathrm{H}(\mathrm{m})$ & $\mathrm{IMAv}\left(\mathrm{m}^{3}\right)$ \\
\hline Spondias venulosa & $32,3 \mathrm{a}$ & $17,5(-)$ & $0,7415(-)$ \\
Cariniana legalis & $18,6 \mathrm{~b}$ & $13,7(-)$ & $0,1648(-)$ \\
Zeyheria tuberculosa & $18,4 \mathrm{~b}$ & $12,3 \mathrm{~b}$ & $0,1987 \mathrm{a}$ \\
Basiloxylon brasiliensis & $17,9 \mathrm{bc}$ & $14,3 \mathrm{a}$ & $0,1796 \mathrm{ab}$ \\
Kielmeyera albopunctata & $17,6 \mathrm{bc}$ & $14,1(-)$ & $0,1678 \mathrm{~b}$ \\
Lecythis pisonis & $16,1 \mathrm{c}$ & $13,4(-)$ & $0,1866(-)$ \\
Astronium concinnum & $15,5 \mathrm{~cd}$ & $13,6 \mathrm{a}$ & $0,1373 \mathrm{c}$ \\
Goniorrhachis marginata & $14,6 \mathrm{~d}$ & $11,9 \mathrm{~b}$ & $0,0989(-)$ \\
Vitex sp. & $13,5 \mathrm{de}$ & $10,4 \mathrm{c}$ & $0,0850 \mathrm{~d}$ \\
Manilkara bella & $12,9 \mathrm{e}$ & $11,7(-)$ & $0,1166 \mathrm{c}$ \\
\hline
\end{tabular}

Médias seguidas pela mesma letra, na coluna, não diferem significativamente entre si, pelo teste $\mathrm{t}$, a $5 \%$ de probabilidade. (-) os dados não apresentaram distribuição normal pelo teste de Lilliefors e, portanto, não foram comparados pelo teste t.

pecuniário no mercado, o suficiente para compensar o lento crescimento das árvores dessas espécies.

Os povoamentos de Zeyheria tuberculosa, Cariniana legalis, Basiloxylon brasiliensis, Kielmeyera albopunctata, Astronium concinnum e Lecythis pisonis, apresentaram valores médios de DAP iguais ou superiores a $15 \mathrm{~cm}$ apenas nas idades de 15,16,17, 17, 17 e 18 anos, respectivamente. Como as dimensões mínimas de corte dessas espécies foram relativamente próximas, a escolha daquela(s) a ser(em) implantada(s) em plantios comerciais dependerá do valor de mercado da madeira de cada espécie.

Considerando um DAP mínimo para utilização em serraria de $20 \mathrm{~cm}$, apenas os povoamentos de Basiloxylon brasiliensis e Spondias venulosa apresentaram DAP médio superior a esse valor no último ano de avaliação (Figura 1). O povoamento de Basiloxylon brasiliensis atingiu DAP superior a $20 \mathrm{~cm}$ após 23 anos de formação, já Spondias venulosa, entre 10 e 11 anos. Isso mostra que o (longo) tempo necessário para se obter o retorno econômico com o plantio das demais espécies avaliadas poderia inviabilizar um investimento.

Drumond et al. (1998) verificaram em plantios de eucalipto com oito anos de idade na região dos Tabuleiros Costeiros, em Sergipe, valores de DAP variando de 8,9 a 9,7 cm para Corymbia citriodora, de 9,3 cm para Eucalyptus grandis e de 9,1 a 10,5 cm para Eucalyptus urophylla. Apenas os povoamentos de Kielmeyera albopunctata, Lecythis pisonis, Zeyheria tuberculosa, Astronium concinnum e Spondias venulosa apresentaram valores de DAP semelhantes ou superiores aos obtidos por esses autores. Dentre essas espécies, destacam-se o Astronium concinnum, com DAP médio de $11 \mathrm{~cm}$, e Spondias venulosa, com DAP de $18 \mathrm{~cm}$, na referida idade. Todavia, é necessário avaliar as características que influenciam no rendimento da produção madeireira, como tortuosidade e bifurcação.

De forma geral, as espécies apresentaram incremento médio anual volumétrico (IMAv) máximo em idades precoces (Figuras 3 e 4). Constata-se que o espaçamento de plantio reduzido antecipou a competição entre os indivíduos e que os desbastes realizados não foram realizados no tempo e em intensidades suficientes para evitar a estagnação do crescimento.

Carvalho $(2003 ; 2006 ; 2010)$ apresentou dados de crescimento de plantios de espécies nativas em diversas idades e em diferentes Estados do Brasil. Zeyheria tuberculosa apresentou IMAv variando de 5,65 a 24,00 $\mathrm{m}^{3} \mathrm{ha}^{-1}$ ano $^{-1}$ e DAP médio variando de 15,1 a 22,4 cm. Cariniana legalis apresentou valores de IMAv variando de 4,30 a 21,70 $\mathrm{m}^{3} \mathrm{ha}^{-1}$ ano $^{-1}$ e DAP médio variando 3,0 a 17,3 cm. Lecythis pisonis 


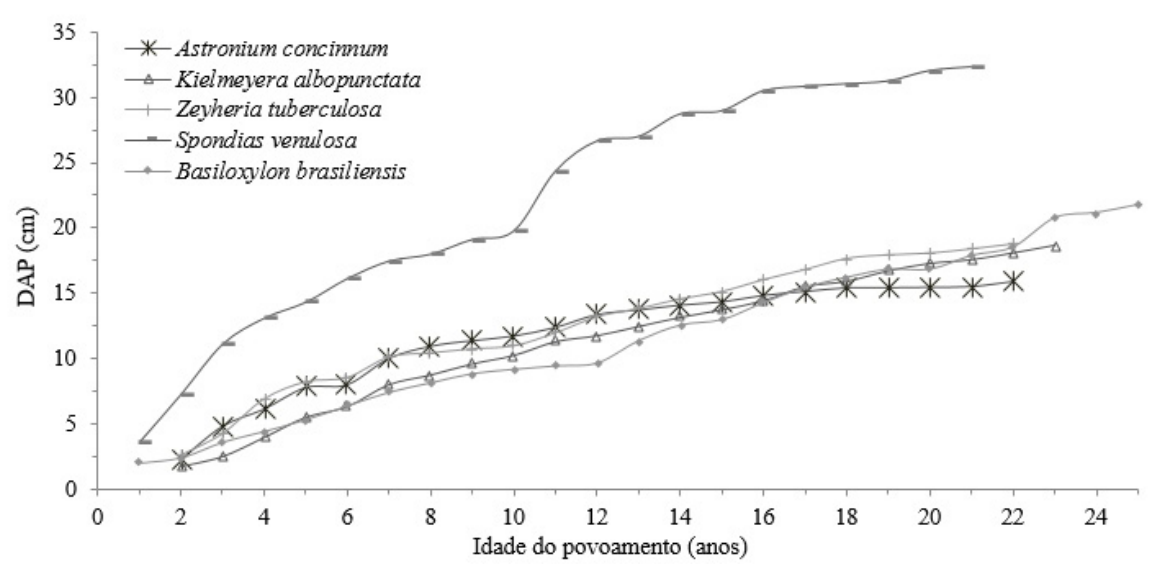

FIGURA 1: Diâmetro a altura do peito (DAP) médio das cinco espécies arbóreas nativas da Mata Atlântica de maior crescimento, implantadas em plantios puros no espaçamento de 2 x 2 metros em Linhares - ES.

FIGURE 1: Diameter at breast height of the average (DAP) of five native tree species of the Atlantic Forest of highest growth, implemented in pure stands with spacing of $2 \times 2$ meters in Linhares, ES state.

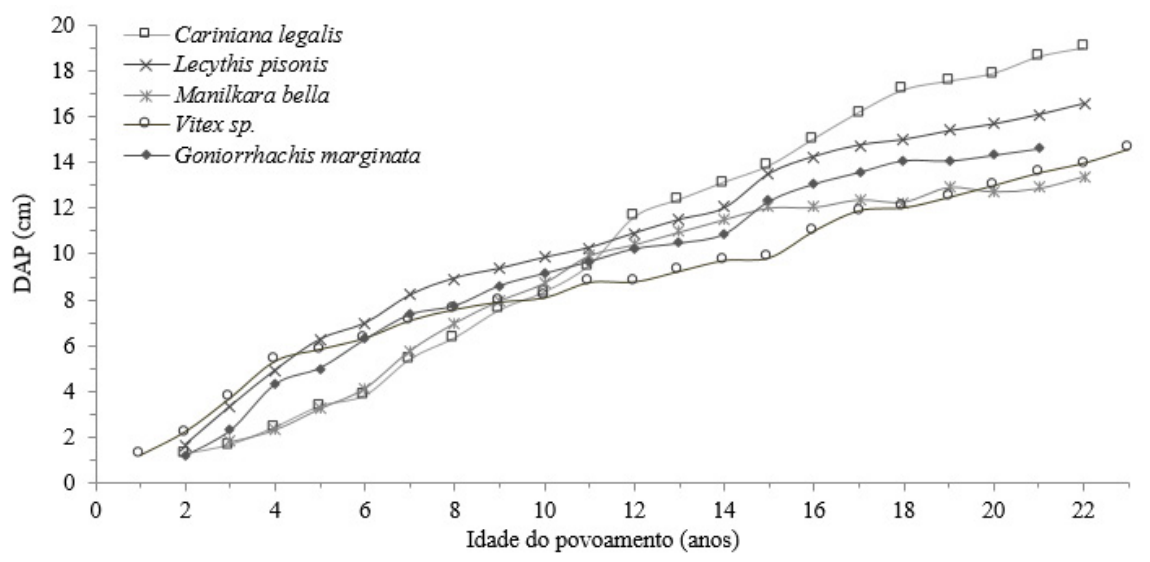

FIGURA 2: Diâmetro a altura do peito (DAP) médio das cinco espécies arbóreas nativas da Mata Atlântica de menor crescimento, implantadas em plantios puros no espaçamento de 2 × 2 metros em Linhares - ES.

FIGURE 2: Diameter at breast height of the average (DAP) of five native tree species of the Atlantic Forest of smaller growth, implemented in pure stands with spacing of $2 \times 2$ meters in Linhares, ES state.

apresentou DAP médio variando de 2,6 a 21,0 cm. Vitex sp. apresentou DAP médio variando de 4,1 a 9,5 $\mathrm{cm}$. Silva (2013) apresentou uma estimativa de DAP de $21 \mathrm{~cm}$ para Cariniana legalis aos 20 anos em sítios de produtividade moderada. Segundo Carpanezzzi (1996), citado por Carvalho (2010), plantios com espécies nativas no sul do país possuem produtividade média anual estimada $\mathrm{em} 8 \mathrm{~m}^{3} \mathrm{ha}^{-1}$ em rotação de 40 anos.

Os baixos crescimentos diamétrico e volumétrico alcançados após o longo período de avaliação para a maioria das espécies são fatores importantes a serem considerados, pois podem inviabilizar plantios comerciais dessas espécies. Nesse caso, o melhoramento genético e a definição de práticas silviculturais mais adequadas visando à produção madeireira, inexistentes para essas espécies, é imprescindível. Contudo, deve-se considerar que os solos de Tabuleiros Costeiros são pobres em nutrientes e possuem 


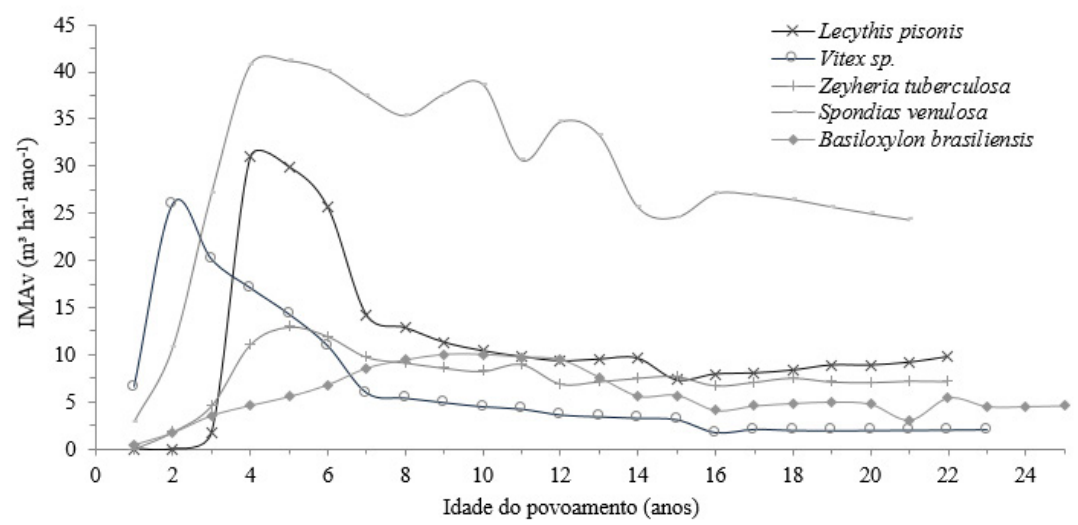

FIGURA 3: Incremento médio anual volumétrico (IMAv) das cinco espécies arbóreas nativas da Mata Atlântica de maior crescimento, implantadas em plantios puros no espaçamento de $2 \times 2$ metros em Linhares - ES.

FIGURE 3: Average annual volume increment (IMAv) of five native tree species of the Atlantic Forest of highest growth, implemented in pure stands with spacing of 2 × 2 meters in Linhares, ES state.

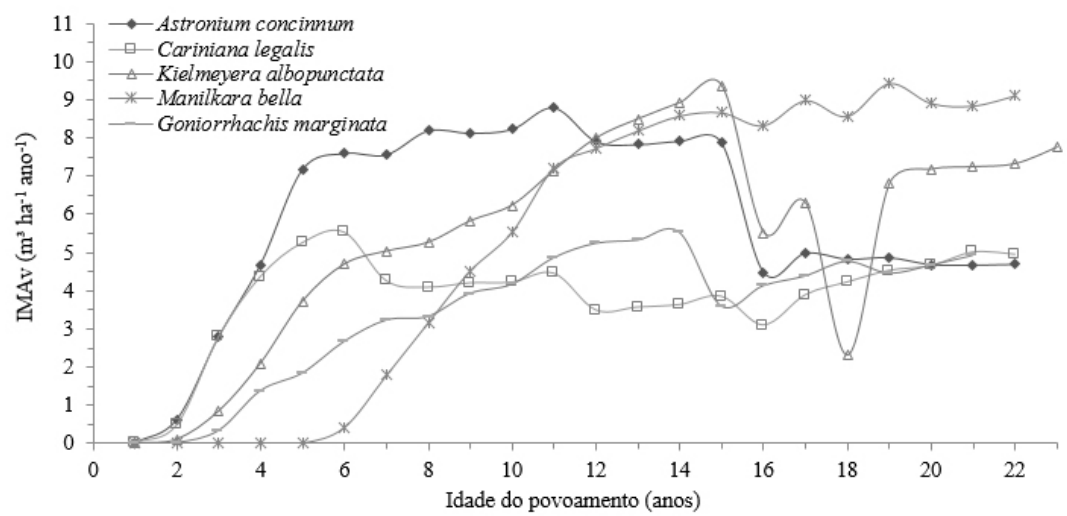

FIGURA 4: Incremento médio anual volumétrico (IMAv) das cinco espécies arbóreas nativas da Mata Atlântica de menor crescimento, implantadas em plantios puros no espaçamento de 2 x 2 metros em Linhares - ES.

FIGURE 4: Average annual volume increment (IMAv) of five native tree species of the Atlantic Forest of smaller growth, implemented in pure stands with spacing of 2 × 2 meters in Linhares, ES state.

horizonte superficial arenoso de baixa capacidade de retenção de nutrientes e água (GARAY et al., 2003; LIMA NETO et al., 2009) e que talvez as práticas corretivas adotadas na época podem não ter amenizado essas deficiências. É provável ainda que a não utilização do critério técnico de desbaste com base no máximo incremento médio anual em volume possa ter influenciado negativamente nos crescimentos apresentados.

Ressalta-se a importância de estudos de tecnologia da madeira que visem identificar os usos mais apropriados, contribuindo, assim, para o melhor aproveitamento dessas e outras espécies nativas. Esses estudos devem também levar em consideração a idade dos indivíduos plantados.

A escolha de uma ou outra espécie para plantios econômicos pode ser também influenciada pela possibilidade de comercialização de produtos florestais não madeireiros, como a comercialização de castanhas e frutos para Lecythis pisonis e Spondias venulosa, respectivamente (INHETVIN, 2010; JUSTINIANO et al., 2001), já que a produção dos mesmos poderia gerar rendimentos periódicos intermediários até se chegar no corte final do povoamento. 


\section{CONCLUSÕES}

Nenhuma espécie estudada apresenta impedimentos à silvicultura. Contudo, algumas apresentam restrições devido a elevadas falhas de plantio (Zeyheria tuberculosa, Goniorrhachis marginata e, especialmente, Cariniana legalis), problemas fitossanitários (Vitex sp.), suscetibilidade à quebra (Spondias venulosa e Goniorrhachis marginata), pelos elevados índices de bifurcação (Kielmeyera albopunctata, Lecythis pisonis, Vitex sp. e Spondias venulosa), o que é agravado quando as ramificações ocorrem em partes mais baixas do tronco, e pela forma tortuosa do fuste (Spondias venulosa. Vitex sp. e Goniorrhachis marginata). Para a produção madeireira, os crescimentos diamétrico e volumétrico apresentados pelas espécies podem ser considerados insatisfatórios, com exceção de Spondias venulosa.

Para o desenvolvimento da silvicultura dessas e de outras espécies nativas, são fundamentais estudos de técnicas silviculturais, melhoramento genético e tecnologia da madeira. Além disso, é necessário aprimorar a tecnologia de desdobro e de beneficiamento de produtos madeireiros visando à utilização de toras de menores diâmetros e a otimização de seu uso.

\section{AGRADECIMENTOS}

Os autores agradecem a empresa Vale S.A. pela disponibilização dos dados e apoio nos trabalhos de campo no âmbito do projeto: "Estudos de Silvicultura Tropical, Ecologia e Manejo Florestal e Recuperação de Áreas Degradadas", por meio de convênio realizado entre a empresa e a Universidade Federal do Espírito Santo/Departamento de Ciências Florestais e da Madeira.

\section{REFERÊNCIAS}

ANDRADE, H. B. Avaliação de espécies e procedências de Eucalyptus L'Héritier (Myrtaceae) nas Regiões Norte e Noroeste do Estado de Minas Gerais. 1991. Dissertação (Mestrado) - Escola Superior de Agricultura de Lavras, Lavras, 1991. AGÊNCIA DE SERVIÇOS PÚBLICOS DE ENERGIA DO ESTADO DO ESPÍRITO SANTO. Vitória:

ASPE, 2012. Disponível em: < http://www.aspe.es.gov.br>. Acesso em: 1 jun. 2012. ASSOCIAÇÃO BRASILEIRA DE PRODUTORES DE FLORESTAS PLANTADAS. Anuário estatístico da ABRAF 2012 ano base 2011. Brasília: ABRAF, 2012. Disponível em:

$<$ http://www.ipef.br/estatisticas/relatorios/anuario-ABRAF12-BR.pdf $>$. Acesso em: 1 out. 2012

BRANCALION, P. H. S. et al. Silvicultura de espécies nativas para viabilização econômica da restauração florestal na Mata Atlântica. In: MARTINS, S. V. (Ed.). Restauração Ecológica de Ecossistemas Degradados. Viçosa: UFV, 2012. cap. 7, p. 212-239.

CARVALHO, P. E. R. Espécies arbóreas brasileiras. Brasília: Embrapa Informação Tecnológica; Colombo: Embrapa Florestas, 2003. v. 1, 1.093 p. (Coleção Espécies Arbóreas Brasileiras).

CARVALHO, P. E. R. Espécies arbóreas brasileiras. Brasília: Embrapa Informação Tecnológica; Colombo: Embrapa Florestas, 2006. v. 2, 627 p. (Coleção Espécies Arbóreas Brasileiras).

CARVALHO, P. E. R. Espécies arbóreas brasileiras. Brasília: Embrapa Informação Tecnológica; Colombo: Embrapa Florestas, 2010. v. 4, 644 p. (Coleção Espécies Arbóreas Brasileiras).

DRUMOND, M. A.; OLIVEIRA, V. R.; CARVALHO, O. M. Comportamento silvicultural de espécies e procedências de Eucalyptus na região dos Tabuleiros Costeiros do Estado do Sergipe. Revista Árvore, Viçosa, v. 22, n. 1, p. 137-142, 1998.

ENGEL, V. L.; POGGIANI, F. Influência do sombreamento sobre o crescimento de mudas de algumas essências nativas e suas implicações ecológicas e silviculturais. IPEF, Piracicaba, n. 43/44, p. 1-10, jan./dez. 1990.

FONTES, M. A. L. Padrões alométricos em espécies arbóreas pioneiras tropicais. Scientia Forestalis. Piracicaba, n. 55, p. 79-87, jun. 1999.

GARAY, I.; KINDEL, A.; JESUS, R. M. Diversity of humus forms in the Atlantic forest ecosystems (Brazil): the table-land Atlantic forest. Acta Oecologica, Philidelphia, n. 16, p. 553-570, 1995.

GARAY, I.; et al. Diversidade funcional dos solos na Floresta Atlântica de Tabuleiros. In: ; RIZZINI, 
C. M. (orgs). A Floresta Atlântica de Tabuleiros: diversidade funcional da cobertura arbórea. Petrópolis: Vozes, 2003. p. 16-26.

GONZAGA, A. L. Madeira: uso e conservação. Brasília: IPHAN/MONUMENTA, 2006. 246 p.

HIROTA, M. M. (Coord.). Atlas dos remanescentes florestais da Mata Atlântica: período 2011-2012. Relatório técnico. São Paulo: [s. n.], 2013. 61 p.

INSTITUTO CAPIXABA DE PESQUISA, ASSISTÊNCIA E EXTENSÃO RURAL. Sistema de informações meteorológicas. Vitória: INCAPER, [2012]. Disponível em: <http://hidrometeorologia. incaper.es.gov.br/>. Acesso em: 1 fev. 2012.

INHETVIN, T. Cadeias de Valor da Sociobiodiversidade no Corredor Central da Mata Atlântica (Bahia e Espírito Santo). Relatório do Estudo. Vitória: Projeto Corredores Ecológicos/ PCE, 2010.

INSTITUTO DE PESQUISAS TECNOLÓGICAS DO ESTADO DE SÃO PAULO. Banco de dados. São Paulo: IPT/SP, 2012. Disponível em:

$<\mathrm{http} / /$ www.ipt.br/consultas_online/informacoes_sobre_madeira/busca $>$. Acesso em: 1 fev. 2012.

JUSTINIANO, M. J.; FREDERICKSEN, T. S.; NASH G. D. Ecologia y silvicultura de especies menos conocidas. Proyecto de manejo forestal sostenible Bolfor. Santa Cruz: Bolívia, 2001.

KAGEYAMA, P. Y.; CASTRO, C. F. A. Sucessão secundária, estrutura genética e plantações de espécies arbóreas nativas. IPEF, Piracicaba, n. 41/42, p. 83-93, jan./dez. 1989.

KINDEL, A.; GARAY, I. Caracterização de ecossistemas da Floresta Atlântica de Tabuleiros por meio das formas de húmus. Revista Brasileira de Ciência do Solo, Viçosa, n. 25, 551-563, 2001.

Serviço Florestal Brasileiro (SFB). Banco de Dados de Espécies de Madeiras Brasileiras. LPF; Serviço Florestal Brasileiro. Disponível em: < http://sistemas.florestal.gov.br/madeirasdobrasil $>$. Acesso em: 1 fev. 2012.

LIMA NETO, J. A. et al. Caracterização e gênese do caráter coeso em Latossolos Amarelos e Argissolos dos Tabuleiros Costeiros do estado de Alagoas. Revista Brasileira de Ciência do Solo, Viçosa, MG, v. 33, n. 4, p. 1001-1011, 2009.

LORENZI, H. Árvores brasileiras: manual de identificação e cultivo de plantas arbóreas nativas do Brasil. 5. ed. Nova Odessa: Instituto Plantarum, 2008. v. 1, 352 p.

LORENZI, H. Árvores brasileiras: manual de identificação e cultivo de plantas arbóreas nativas do Brasil. 3. ed. Nova Odessa: Instituto Plantarum, 2009. v. 2, 384 p.

MARTINS, E. P.; OLIVEIRA, A. D.; SCOLFORO, J. R. S. Avaliação dos danos causados pela exploração florestal à vegetação remanescente em florestas naturais. Cerne, Lavras, v. 3. n. 1, p. 14-24, 1997.

MARTINS, L. T. Caracterização dendrométrica e crescimento de espécies não tradicionais em plantios homogêneos. 2012. Dissertação (Mestrado) - Universidade Federal do Espírito Santo, Jerônimo Monteiro, 2012.

MATTOS, R. B.; DURLO, M. A.; LÚCIO, A. L. D. Possibilidade de ganho de fuste em espécies euxilóforas nativas da região central do Rio Grande do Sul. Ciência Florestal, Santa Maria, v. 13, n. 2, p. 11-120, dez. 2003.

MARCHESAN, R. Rendimento e qualidade de madeira serrada de três espécies tropicais. Dissertação (mestrado) - Universidade Federal do Paraná, Curitiba, 2012.

OGAYA, N. Kubierungsformeln und Bestandesmassenformeln. 1968. 85 f. Thesis (PhD) - Universität Freiburg, Breisgau, 1968.

ROCHA, M. P. Técnicas de planejamento em serrarias. Curitiba: FUPEF, 2002. 121 p. (Série Didática FUPEF).

SCHILLING, A. C. et al. Influência de diferentes intensidades de desrama sobre a porcentagem de lenho tardio e quantidade de nós da madeira de primeiro desbaste de Pinus elliottii Engelman. Ciência Florestal, Santa Maria, v. 8, n. 1, 1998.

SCHUMACHER, F. X.; HALL, F. S. Logarithmic expression of timber-tree volume. Journal of Agricultural Research, Washington, v. 47, n. 9, p. 719-734, 1933.

SILVA, C. C. Potencial de espécies nativas para a produção de madeira serrada em plantios de restauração florestal. 2013. Dissertação (Mestrado) - Escola Superior de Agricultura "Luiz de Queiroz", Lavras, 2013.

SPURR, S. H. Forest inventory. New York: Ronald Press, 1952. 
StOATE, T. N. The use of a volume equation in pine stands. Australian Forestry, Queen Victoria, v. 9, p. 48-52, 1945.

TANAKA, A.; VIEIRA, G. Autoecologia das espécies florestais em regime de plantio de enriquecimento em linha na floresta primária da Amazônia Central. Acta Amazonica, Manaus, v. 36, n. 2, p. 193-204, 2006. VELOSO, H. P.; RANGEL FILHO, A. L. R.; LIMA J. C. A. Classificação da vegetação brasileira adaptada a um sistema universal. Rio de Janeiro: IBGE, 1991. 123 p.

YOUNG, C. E. F. Economia do Extrativismo em Áreas de Mata Atlântica. In: SIMÕES, L. P.; LINO, C. F. (Org.). Sustentável Mata Atlântica: a exploração de seus recursos florestais. 2. ed. São Paulo: SENAC, 2003.

WHATleY, J. M.; WHATleY, F. R. A luz e a vida das plantas. São Paulo: EPU/EDUSP, 1982. 101 p. (Temas de Biologia, 30). 